\title{
Fieldable Digital Coherent Interferometric Communication and Sensing Application Domains
}

\author{
I. Shpantzer \\ CeLight, Inc., 12200 Tech Road, Suite 300, Silver Spring, Maryland 20904
}

\begin{abstract}
A layered architecture unifying optical coherent communications and interferometric sensing via digitally stabilized quadrature modulator and homodyne receiver augmented by digital noise reduction and channel compensation algorithms stacks, form a new paradigm for coherent fieldable applications.

(C)2006 Optical Society of America

OCIS codes: (060.1660) Coherent communications; 280.3420 Laser sensors; (130.3120) Integrated optics devices
\end{abstract}

\section{Introduction}

To recapture the benefits of DSP established in a variety of RF application domains in the last decades one has to be able to operate on the optical field vector as opposed to the optical energy. This approach enables deployment of digital coherent communications and interferometric sensing applications but requires overcoming a myriad of technological and architectural challenges of which their proposed solutions are described in this paper. In [1], we discussed the key methods in secure digital coherent free-space optical communications for tactical applications.

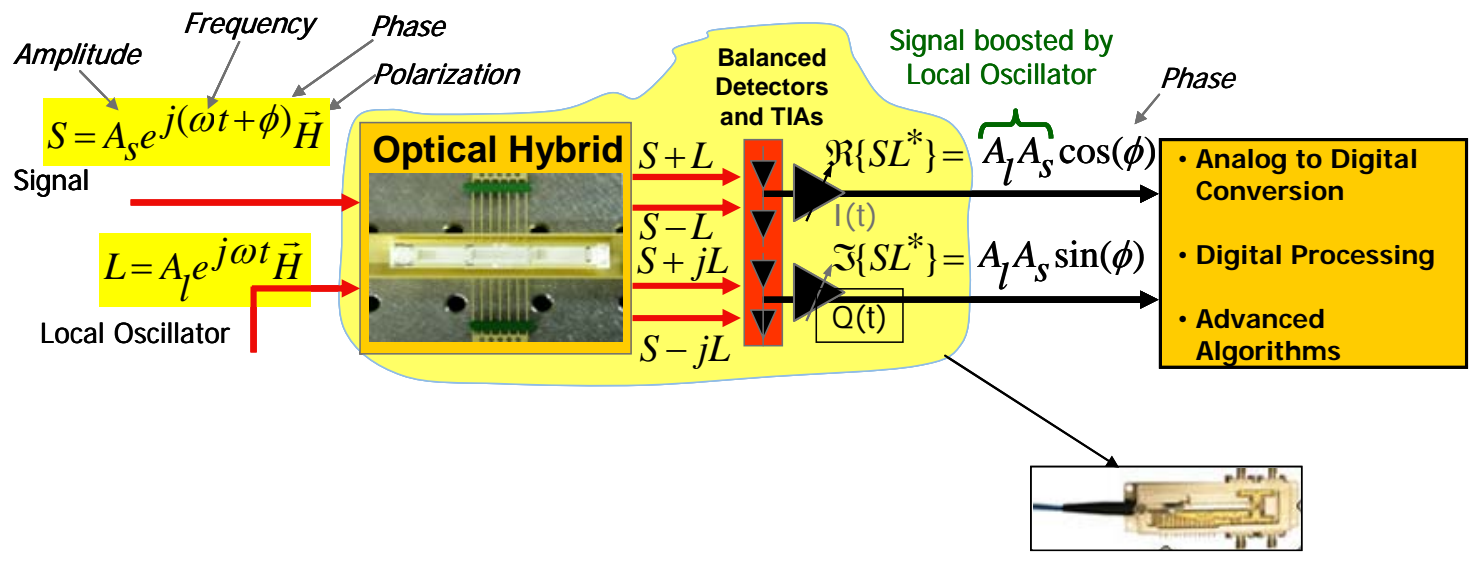

Fig. 1. Digital coherent integrated Homodyne Receiver for linear transformation of an encoded optical signal to base-band. Top view shows a picture of the fabricated and a tested device is shown on bottom right. Only one polarization is shown for simplicity

Fig. 1 depicts an integrated Homodyne Receiver we fabricated and tested. The received optical signal is split into two (arbitrary) orthogonal polarizations ( $\mathrm{H}^{\prime}$ and $\mathrm{V}^{\prime}$ were only one is shown for clarity) and each is mixed with a local oscillator in an optical $90^{\circ}$ hybrid. The hybrid accepts the signal S and the local oscillator $\mathrm{L}$ and produces four outputs: (i) $S+L$, (ii) $S-L$, (iii) $S+j L$ and (iv) $S-j L$. Each of the optical output pairs (i)-(ii) and (iii)-(iv) is collected by a pair of matched photodiodes whose photocurrents are subtracted to produce output currents proportional to $|S+L|^{2}-|S-L|^{2}=4 \cdot \operatorname{Re}\left\{S L^{*}\right\}$ and $|S+j L|^{2}-|S-j L|^{2}=4 \cdot \operatorname{Im}\left\{S L^{*}\right\}$, together constructing the complex value $S L^{*}$. Following this linear transformation the signals are electrically filtered, sampled and then processed digitally as described in [1] .

The key advantages of this coherent detection scheme are: (i) The received signal is boosted by the local oscillator for highest shot-noise limited receiver sensitivity; (ii) An inherently frequency-selective receiver using an agile local oscillator; and (iii) Linear down-conversion to electrical baseband which enables adaptive DSP-based noise reduction algorithms. The linear transformation allows for changing the order of compensation thus enabling backend digital adaptive algorithms to compensate for a variety of noise sources thus eliminating traditional complex "front-end" optical techniques such as optical phase locking and polarization compensation. Furthermore, adaptive stabilization of the interferometric components to maintain their operational points is solved as described in detail in [2] and further discussed in Sec. 3.

The key benefit of the linear transformation described above results from the ability to operate on the field vector thus enabling the digital adaptive compensation of various phase noise induced by physical phenomena such as: (i) Platform vibrations; (ii) Doppler shift; (iii) Polarization rotation and birefringence, (iv) High speed air turbulence 


\section{CWC1.pdf}

induced fading and scintillation that cannot be compensated by adaptive optics; and (v) Electronic beam-steering. These advantages are discussed in [1] and in Sec. 4.

\section{Layered Architecture for Digital Coherent Communications and Sensing}

A unified layered architecture for fieldable digital coherent interferometric communications and sensing is depicted in Fig. 1. The layered architecture is based on two integrated optical components that enable the embodiment of a generalized transponder consisting of Synthesizer and Analyzer constructs each consisting of three layers: (i) An Optical layer, composed of an integrated Quadrature Modulator and a Homodyne Receiver that performs the linear transformation of coherent optical signals to/from electrical base-band; (ii) Stabilization layer that maintains the optical components at an optimized operating point; and (iii) Adaptive DSP-based noise cancellation to compensate for multiplicative phase noise resulting from platform vibration, Doppler shift, polarization rotation, fading and scintillation as detailed in Sec. 4 and [1].

\section{Coherent Applications}

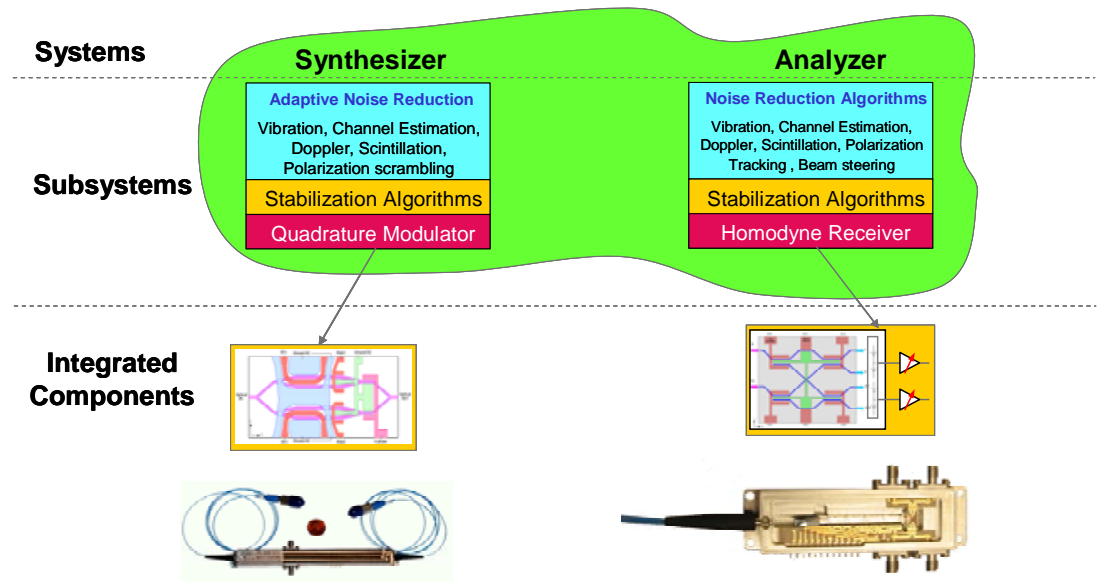

Fig. 2. A unified layered architecture for fieldable digital coherent interferometric communications and sensing.

In the case of free space optical communications the Synthesizer provides: Agile synthesis of key-based multi dimensional hopping in time, frequency, polarization state, coherent modulation scheme (e.g. M-PSK, M-QAM) and symbol rate to adapt to an optimal combination of security against jamming and eavesdropping, spectral efficiency and atmospheric conditions in the tactical environment.

In the latter application the Analyzer embodies: Coherent detection of a generalized key-based multi-dimensionally hopped coherent optical signal via a generalized Homodyne Polarization Diversity Receiver with DSP-based adaptive algorithms that digitally extract the information content from both channel noise and key-based multi dimensional optical scrambling without the use of optical frequency and polarization tracking and unwinding. Furthermore, for improved security and flexibility, this approach does not require a fixed DWDM-like channel structure, but instead takes advantage of contiguous gridless selection of any optical carrier frequency based on the atmospheric conditions and tactical operational needs as described in [1]. High spectral efficiency fiber based coherent communications utilizing our components are described in [3], [4] and an OCDMA approach in [5].

By co-locating the synthesizer and analyzer and sharing a common optical local oscillator one can design interferometric sensing applications such as Coherent LADAR or Vibrometery using a common layered architecture with the unique ability to switch applications via software control.

\section{Stabilization of Integrated Interferometric Optical Components}

Both interferometric components were designed, fabricated and tested with closed loop stabilization of their operating point for uninterrupted up/down linear conversion of base-band electrical signals of up to 12.5 GSymbols/s. A stabilization algorithm for our Quadrature Modulator (QM) is described in [2] and shown in Fig. 3. Both simulation and experimental results for generation of 12.5 GSym/s of QPSK signals are described in [2]. The stabilization algorithm shows a 1-dB sensitivity penalty compared with manual adjustment of the QM via minimization of the BER. Similar stabilization algorithms were developed and tested for the Homodyne Receiver and will be reported at the conference. Further improvement of stabilization algorithms to accommodate interferometric sensing is planned. 


\section{CWC1.pdf}
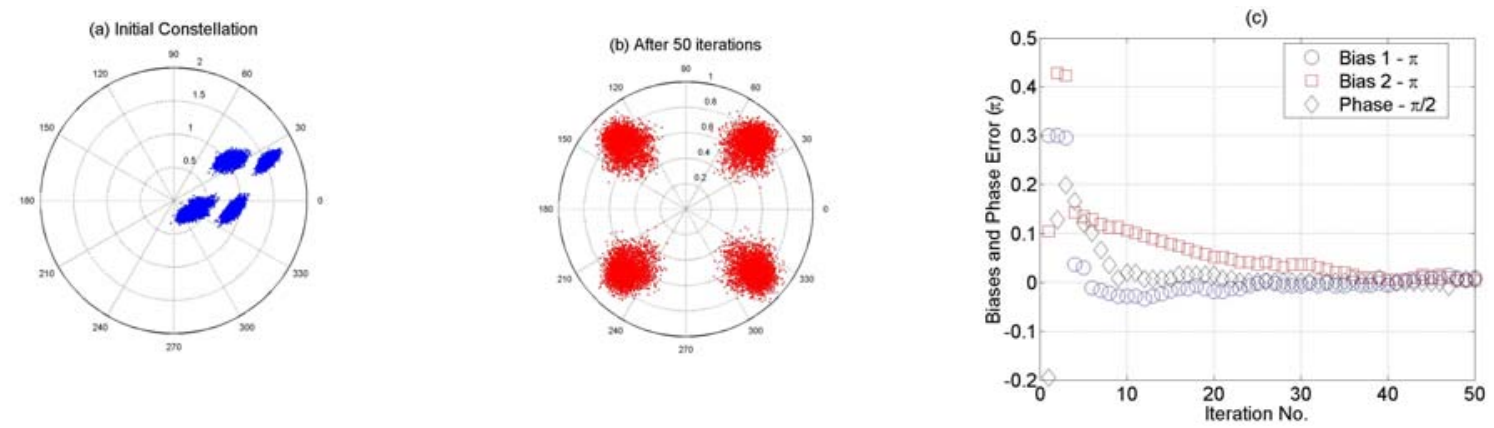

Fig. 3. Constellation plots of the QM optical output at startup (a) and after 50 iterations of the control loop (b). Plot of deviations of the two biases and phase from their optimal points ( $\pi$ and $\pi / 2$ ) versus iteration number are shown in (c).

\section{Adaptive Noise Reduction Algorithms}

In [1] we discussed digital adaptive noise reduction algorithms for compensation of various physical phenomena. Fig. 4(a) shows schematically the architectural approach as applied to channel compensation of air turbulence for free space coherent QPSK communications. Fig. 4(b) shows simulated results of a free space QPSK optical link operating over a high speed turbulent channel before and after adaptive noise reduction as well as the time convergence track.

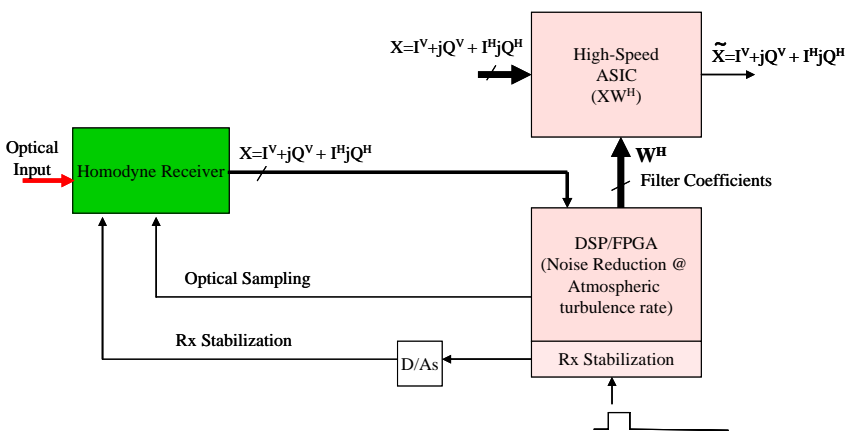

(a)

Fig. 4(a) Digital adaptive algorithms embodiment; and (b) Performance of the channel equalization algorithm over a turbulent atmospheric channel.

\section{Interferometric Sensing}

By co-locating the synthesizer and analyzer with an optical local oscillator derived from the transmitting laser, the transponder configuration turns into an interferometric sensing system enabling application domains such as Coherent LADAR and Vibrometery, and remote chemical detection; all using a unified layered architecture with the unique ability to adapt, reconfigure, and switch coherent applications via software control.

\section{Acknowledgements}

CeLight team: P. Cho, A. Kaplan, Y. Achiam, S. Kazi, A. Greenblatt, G. Harston, J. Khurgin, and A. Salamon,

\section{References}

[1] A Salamon, G. Yurista, M. Tseytlin, P. S. Cho, I. Shpantzer, "Secure optical communications utilizing PSK modulation, polarization multiplexing and coherent digital homodyne detection with wavelength and polarization agility,” MILCOM 2003, Boston, MA, Oct. 13-16, U026.

[2] P. S. Cho, J. B. Khurgin, and I. Shpantzer, "Closed-loop control of LiNbO3 quadrature modulator for coherent communications", COTA Conference Proceedings, Whistler Canada, June 2006.

[3] P. S. Cho, G. Harston, C. Kerr, A. Greenblatt, A. Kaplan, Y. Achiam, and I. Shpantzer, "Coherent homodyne detection of BPSK signals using time-gated amplification and $\mathrm{LiNbO}_{3}$ optical $90^{\circ}$ hybrid,” IEEE Photonics Technology Letters, vol. 16, no. 7, pp. 17271729, July, 2004.

[4] P. S. Cho, et al., "Investigation of 2-bit/s/Hz 40-Gb/s DWDM transmission over 4×100-km SMF-28 fiber using RZ-DQPSK and polarization multiplexing,” Photon. Technol. Lett., 16, 656-658, July, 2004.

[5] J. B. Khurgin, A. B. Cooper III, P. S. Cho, and I. Shpantzer, "Painless fully orthogonal coherent OCDM", COTA Conference Proceedings, Whistler Canada, June 2006. 